CORRIGENDUM

doi:10.1038/nature1 1848

\title{
Corrigendum: Global quantification of mammalian gene expression control
}

Björn Schwanhäusser, Dorothea Busse, Na Li, Gunnar Dittmar, Johannes Schuchhardt, Jana Wolf, Wei Chen \& Matthias Selbach

Nature 473, 337-342 (2011); doi:10.1038/nature10098

Mark Biggin of the Lawrence Berkeley National Laboratory contacted us, noting that our mass-spectrometry-based protein copy number estimates are lower than several literature-based values. We therefore re-analysed the scripts used for data processing, and found a scaling error that occurred during the conversion of normalized protein intensity values into absolute copy number estimates. As described in the original Article, slope and offset for scaling were calculated by linear regression based on an in-solution digest with spiked-in
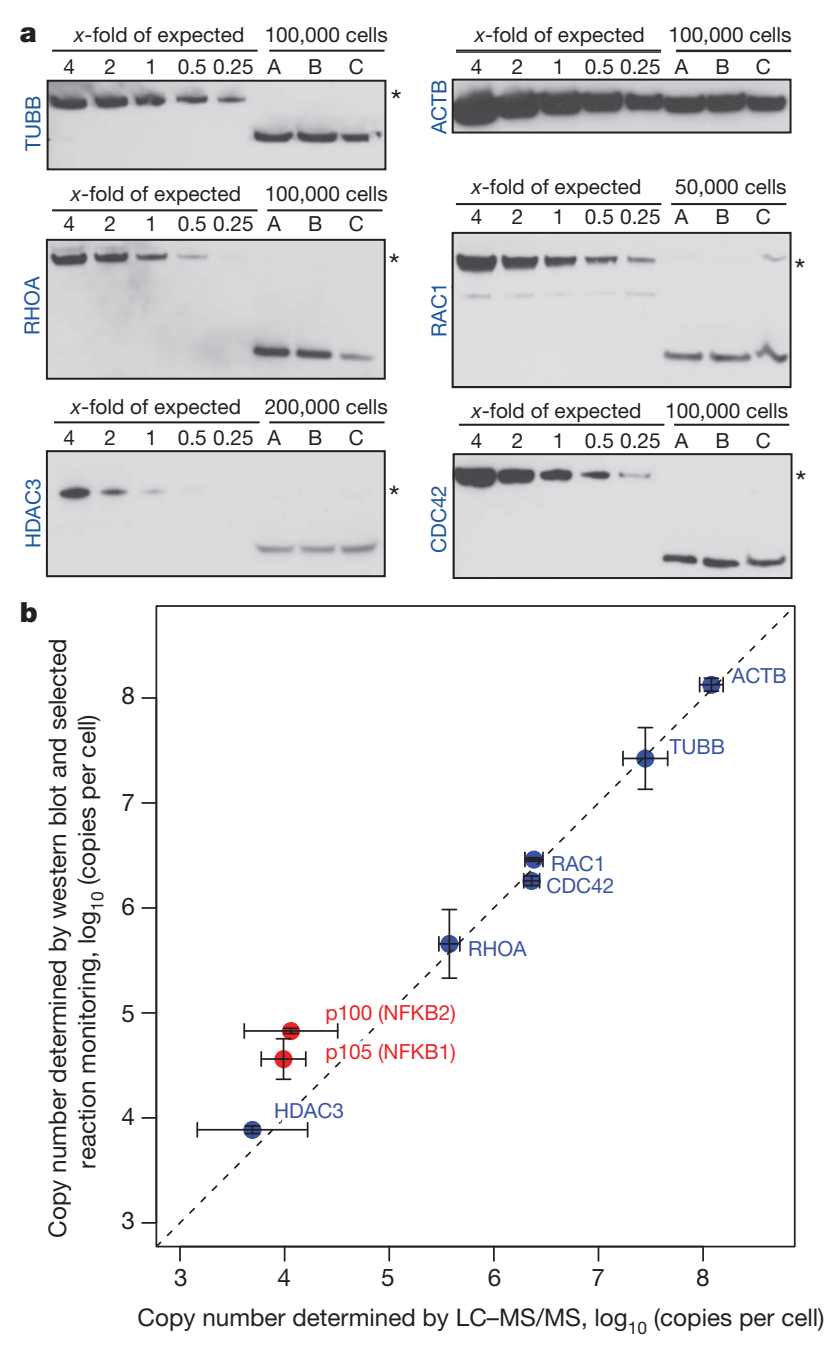

Figure 1 Comparison of LC-MS/MS-based protein copy number estimates in NIH3T3 cells with alternative methods. a, Representative western blots of cellular proteins with dilution series of purified protein standards. Standards were diluted in a way that one-fold corresponds to the amount expected from the average of the LC-MS/MS-based estimates. The asterisks indicate the position of the GST-fusion proteins. $\mathbf{b}$, Comparison of estimates based on western blots (blue, $n \geq 3$ ) and selected reaction monitoring (red, $n=3$ ) with our LC-MS/MS data $(n=2)$. Error bars show standard deviations. proteins of known concentrations. We erroneously used the slope and the offset from an unrelated experiment to scale protein levels, resulting in a systematic underestimation of protein levels and derived translation rate constants. We apologize for this error and any confusion it may have caused.

When the error was corrected, the median levels of detected proteins increased about threefold and the ratio of average protein to messenger RNA increased from 900 to 2,800 . The median and apparent maximum translation rate constants increased from 40 to 140 and from 180 to 1,000 proteins per mRNA per hour, respectively. Consequently, the estimated maximum translation rate constant in sea urchin embryos at $15^{\circ} \mathrm{C}$ ( 140 proteins per mRNA per hour) is lower than our corrected prediction for mouse fibroblasts $(1,000$ proteins per mRNA per hour). All our conclusions about global gene expression control (correlations between mRNA and protein levels and half-lives, predominant control of protein abundance at the level of translation, functional properties of genes with specific half-life

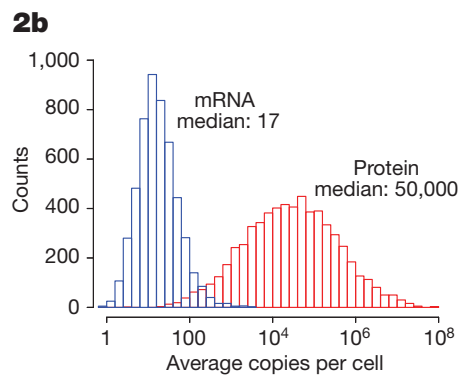

3c
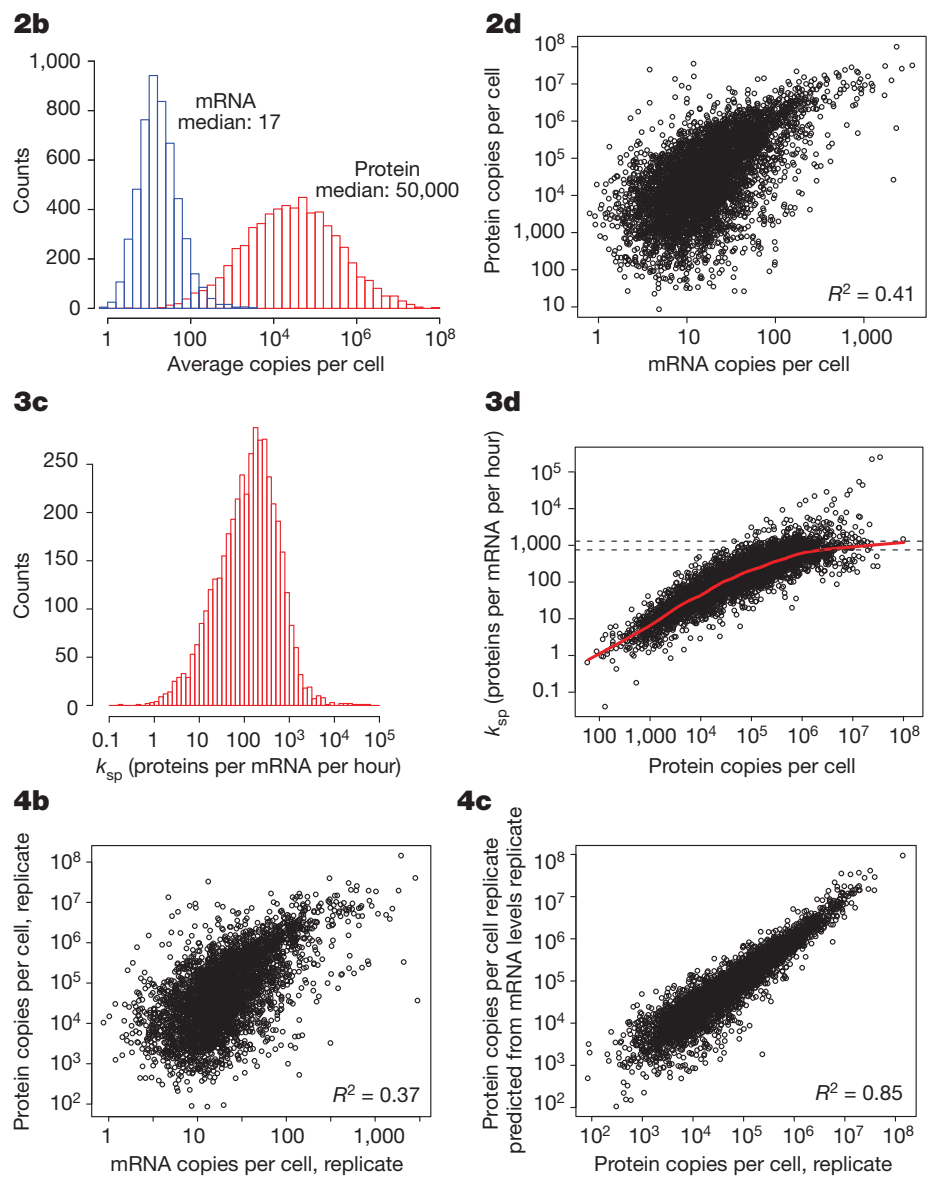

Figure $2 \mid$ This figure shows the corrected panels for Figs $2 b$ and $d, 3 c$ and $d$ and $4 \mathrm{~b}$ and $\mathrm{c}$ of the original Article. We note that although the distribution of data in the original and corrected figures appears very similar, the axes are different. 
combinations and so on) are unaffected. Figure 2 of this Corrigendum shows the corrected Figs $2 \mathrm{~b}$ and d, $3 \mathrm{c}$ and $\mathrm{d}$ and $4 \mathrm{~b}$ and c. Supplementary Figs 5a and b, 6d and f, 8f, 12a and b, and Supplementary Tables 1 and 3 of the original Article have been corrected. Protein copy numbers and translation rate constants in the text and figures in the HTML and PDF versions of the original Article have been corrected.

To further validate copy numbers in NIH3T3 cells we performed western blots with a dilution series of purified human proteins as standards (Fig. 1a of this Corrigendum). Briefly, cells were washed, harvested by trypsinization, counted independently by two persons, lysed in radioimmunoprecipitation assay buffer (containing 1\% SDS) and separated by SDS-polyacrylamide gel electrophoresis (PAGE). As standards, defined amounts of human glutathione-S-transferase (GST)-tagged HDAC3, TUBB (Abnova), RHOA, RAC1 or CDC42 (purified in house and quantified spectrophotometrically) or purified ACTB (Biotrend) were diluted in SDS sample buffer containing $0.07 \mu \mathrm{g}$ Escherichia coli lysate per microlitre to minimize protein loss during dilution. Antibodies against HDAC3 (2632), CDC42 (2466) and Rac1/2/3 (2467) were from Cell Signalling; the ACTB (A5441) and TUBB (T8328) antibodies were from Sigma and the anti-RHOA antibody (SC-418) was from Santa Cruz. Protein abundance in NIH3T3 cells was estimated densitometrically, based on the dilution series as a standard curve (Scion Image). We also used selected reaction monitoring to quantify two additional proteins (p100 and p105). To this end, cells were lysed (6 M urea, $2 \mathrm{M}$ thiourea) and lysates mixed with synthetic-stable-isotope-labelled proteotypic peptides (SpikeTides, JPT Peptide Technologies). Samples were digested and analysed on a Q-Trap 5500 system (AB Sciex) in three technical replicates monitoring three transitions per peptide. Quantification was performed using Multiquant 1.2 (AB Sciex) based on the two most intense transitions. Overall, copy number estimates of the eight proteins obtained by alternative approaches correlated well with our data derived from liquid chromatography and tandem mass spectrometry (LC-MS/MS) (Fig. 1b of this Corrigendum), even though the two measurements based on selected reaction monitoring lie above the diagonal. The data are in good agreement with the expected precision and reproducibility of our large-scale absolute protein quantification approach (see Supplementary Figs $6 \mathrm{~d}$ and $8 \mathrm{~b}$ of the original Article). 